\title{
TRANSMITTER DIVERSITY FOR BINARY PSK MOBILE RADIO
}

\author{
Ahmed Elosmany ${ }^{*}$
}

\begin{abstract}
To combat the effects of multipath fading in mobile radio, a transmitter diversity scheme for binary PSK is investigated. In the transmitter, three carriers with uniform separation equal to the bit rate and three separate antennas are used.
\end{abstract}

\section{INTRODUCTION}

Digital modulation schemes have become of interest in UHF and microwave radio communications [1]. Diversity is an effective technique for combating multipath Rayleigh fading encountered in these channels [2]-[4]. Transmitter diversity with no feedback control loops is suitable for transmission systems requiring simplified receiver structure [5].

In this paper, a transmitter diversity scheme employing binary PSK modulation is considered. In this scheme, three PSK signals, with the same binary information but different carrier frequencies, are transmitted from separate antennas.

\section{SYSTEM MODEL. DESCRIPTION}

Consider a binary PSK system in which the transmitter has three branches as shown in Fig. 1. The input binary data ( \pm 1$)$ is used to modulate simultaneously three carriers $\left(\omega_{c}\right.$ and $\left.\omega_{C} \pm \Delta \omega\right)$ and the modulated carriers are transmitted from separate antennas through multipath channels.

Within any signaling interval of length $T$, the receiver composite input signal and its components can be represented as follows:

* Department of ACG, MTC, Cairo 


$$
\begin{gathered}
r(t)=r_{c}(t)+n(t) \\
r_{c}(t)=r_{1}(t)+r_{2}(t)+r_{3}(t) \\
=A_{C}(t) \cos x(t) \\
r_{1}(t)=\rho_{1} A \cos \left(\omega_{c} t+\phi_{B}+\theta_{1}\right) \\
r_{2}(t)=\rho_{2} k A \cos \left[\left(\omega_{c}+\Delta \omega\right) t+\phi_{s}+\theta_{2}\right] \\
r_{3}(t)=\rho_{3} k A \cos \left[\left(\omega_{C}-\Delta \omega\right) t+\phi_{B}+\theta_{3}\right] \\
x(t)=\omega_{C} t+\phi_{s}+\psi_{c}(t)
\end{gathered}
$$

$A_{C}(t)=A\left[\rho_{1}^{2}+k^{2}\left(\rho_{2}^{2}+\rho_{3}^{2}\right)+2 \rho_{1} \rho_{2} k \cos \left(\Delta \omega t+\theta_{2}-\theta_{1}\right)\right.$

$\left.+2 \rho_{1} \rho_{3} k \cos \left(-\Delta \omega t+\theta_{3}-\theta_{1}\right)+2 \rho_{2} \rho_{3} k^{2} \cos \left(2 \Delta \omega t+\theta_{2}-\theta_{3}\right)\right]^{1 / 2}$

$\psi_{c}(t)=\arctan \left[\frac{\rho_{1} \sin \theta_{1}+\rho_{2} \operatorname{ksin}\left(\Delta \omega t+\theta_{2}\right)+\rho_{3} k \sin \left(-\Delta \omega t+\theta_{3}\right)}{\rho_{1} \cos \theta_{1}+\rho_{2} k \cos \left(\Delta \omega t+\theta_{2}\right)+\rho_{3} k \cos \left(-\Delta \omega t+\theta_{3}\right)}\right]$

$n(t)$ is the additive white Gaussian zero-mean noise with single sided power spectral density $\mathrm{N}_{0} \cdot \phi_{\mathrm{g}}(0$ or $\pi)$ is the carrier phase modulation caused by the input data. $\rho_{1}, \rho_{2}$, and $\rho_{3}$ are statistically independent identically distributed random processes assumed constant (slowly varying) over the considered signaling interval $\mathrm{T}$ each with Rayleigh probability density function (pdf). $\theta_{1}, \theta_{2}$, and $\theta_{3}$ are statistically independent identically distributed random processes assumed constant (slowly varying) over the considered signaling interval each with uniform pdf over a range of $2 \pi$. The factor $k$ controls the transmitter power distribution between the central and the two side carriers.

The carrier recovery can be performed using a squarer followed by a band pass filter and divider as shown in Fig. 1(c). If we neglect nolse the squarer output will be

$$
\begin{aligned}
r_{c}^{2}(t) & =A_{c}^{2}(t) \cos ^{2} x(t) \\
& =0.5 A_{C}^{2}(t)\left\{1+\cos \left[2 \omega_{c} t+2 \psi_{c}(t)\right]\right\}
\end{aligned}
$$

since $2 \phi_{\mathrm{B}}=0$ or $2 \pi$. The BPF passes only the double frequency component and the divider output can be written in the form

$$
u_{\text {ref }}(t)=2 \cos \left[\omega_{c} t+\psi_{c}(t)\right]
$$




\section{BER PERFORMANCE ANALYSIS}

The decision variable is given by

where

$$
\begin{aligned}
v & =\int_{0}^{T} r(t) u_{r e f}(t) d t \\
& =v_{s}+v_{n}
\end{aligned}
$$

$$
\begin{gathered}
v_{B}=\int_{0}^{T} A_{C}(t) \cos \left[\omega_{C} t+\phi_{B}+\psi_{C}(t)\right] 2 \cos \left[\omega_{C} t+\psi_{C}(t)\right] d t \\
=a A T \text { if } \omega_{C} T=2 m \pi \quad(m \text { integer }) \text { and } \Delta \omega T=2 \pi \\
a^{2}=\rho_{1}^{2}+k^{2}\left(\rho_{2}^{2}+\rho_{3}^{2}\right) \\
v_{n}=\int_{0}^{T} n(t) 2 \cos \left[\omega_{C} t+\psi_{c}(t)\right] d t
\end{gathered}
$$

$v_{n}$ is a Gaussian variable with zero mean and variance $N_{0} T$.

The bit error rate (BER) is given by

$$
\begin{aligned}
P_{e} & =P x\left(v<0 / \phi_{B}=0\right) \\
& =0.5 \operatorname{erfc}\left(\sqrt{\gamma_{b}}\right)
\end{aligned}
$$

where

$$
\gamma_{b}=a^{2} E_{b} / N_{0}, \quad E_{b}=A^{2} T / 2
$$

Let us define

$$
z_{1}=\rho_{1}^{2}, z_{2}=k^{2} \rho_{2}^{2}, z_{3}=k^{2} \rho_{3}^{2}, z=z_{1}+z_{2}+z_{3}=\alpha^{2} \geq 0
$$

'he pdf's of $Z_{1}, Z_{2}$, and $z_{3}$ are as follows

$$
\begin{gathered}
\mathrm{p}_{\mathrm{z}_{1}}\left(z_{1}\right)=\left(1 / \overline{\rho^{2}}\right) \exp \left(-\mathrm{z}_{1} / \overline{\rho^{2}}, \mathrm{z}_{1} \geq 0\right. \\
\mathrm{p}_{\mathrm{Z}_{2}}\left(\mathrm{z}_{2}\right)=\left(1 / \mathrm{k}^{2} \overline{\rho^{2}}\right) \exp \left(-\mathrm{z}_{2} / \mathrm{k}^{2} \rho^{\overline{2}}\right), \quad z_{2} \geq 0 \\
\mathrm{p}_{\mathrm{z}_{3}}\left(\mathrm{z}_{3}\right)=\left(1 / \mathrm{k}^{2} \rho^{\overline{2}}\right) \exp \left(-\mathrm{z}_{3} / \mathrm{k}^{2} \rho^{2}\right), \quad z_{3} \geq 0
\end{gathered}
$$

where $\overline{\rho^{2}}=\overline{\rho_{1}^{2}}=\rho_{2}^{\overline{2}}=\rho_{3}^{\overline{2}} \cdot \mathrm{Z}_{1}, \mathrm{Z}_{2}$, and $\mathrm{Z}_{3}$ are independent random variables and $\gamma_{\mathrm{b}}=\mathrm{Z} \mathrm{E} \mathrm{E}_{\mathrm{b}}, \mathrm{N}_{\mathrm{o}} \geq 0$, hence we can get the pdf of $\gamma_{\mathrm{b}}$ 
in the following form :

$$
\begin{gathered}
p\left(\gamma_{b}\right)=\left(1 / \gamma_{c}\right) \exp \left(-\gamma_{b} / \gamma_{c}\right), k=0 \\
p\left(\gamma_{b}\right)=\left[1 / \gamma_{c}\left(1-k^{2}\right)^{2}\right]\left[e^{-\gamma_{b} / \gamma_{c}}-e^{-\gamma_{b} / k^{2} \gamma_{c}}-\left(1-k^{2}\right)\left(\frac{\gamma_{b}}{k^{2} \gamma_{c}} e^{-\gamma_{b} / k^{2} \gamma_{c}}\right],\right. \\
p\left(\gamma_{b}\right)=\left(1 / 2 \gamma_{c}\right)\left(\gamma_{b} / \gamma_{c}\right)^{2} \exp \left(-\gamma_{b} / \gamma_{c}\right), k=1
\end{gathered}
$$

where

$$
\gamma_{c}=\bar{\rho}^{2} E_{b} / N_{0}
$$

The average BER is then given by

$$
\begin{gathered}
\overline{P_{e}}=\int_{0}^{\infty} 0.5 \operatorname{erfc}\left[\sqrt{\gamma_{b}}\right] p\left(\gamma_{b}\right) d \gamma_{b} \\
=0.5\left[1-\sqrt{\gamma_{c}^{\prime}\left(1+\gamma_{c}\right)}\right], k=0 \\
=0.5\left[1-\frac{1}{\left(1-k^{2}\right)^{2}} \sqrt{\gamma_{c}^{\prime}\left(1+\gamma_{c}\right)}+\frac{k^{2}\left(2-k^{2}\right)}{\left(1-k^{2}\right)^{2}} \sqrt{k^{2} \gamma_{c} /\left(1+k^{2} \gamma_{c}\right)}\right. \\
\left.+0.5\left[\frac{k^{2}}{1-k^{2}}\right]\left[\frac{1}{1+k^{2} \gamma_{c}}\right] \sqrt{k^{2} \gamma_{c} /\left(1+k^{2} \gamma_{c}\right)}\right], k \neq 0, k \neq 1 \\
=0.5\left\{1-\sqrt{\gamma_{c} /\left(1+\gamma_{c}\right)}\left[1+0.5\left(1+\gamma_{c}\right)^{-1}+0.375\left(1+\gamma_{c}\right)^{-2}\right]\right\}, k=1
\end{gathered}
$$

Fig. 2 shows the dependence of the average BER on the average input carrier-to-noise ratio (CNR) given by

$$
\gamma_{\text {in }}=\gamma_{c}\left(1+2 k^{2}\right)
$$

for different values of $\mathrm{k}$. The best performance is obtained when the available transmitter power is divided equally between the three carriers (i.e., $k=1$ ). The diversity gain for a BER of $10^{-3}$, defined as the CNR difference between a diversity and non-diversity case, is about $12.5 \mathrm{~dB}$ (for $\mathrm{k}=1$ ). The diversity gain increases by $7 \mathrm{~dB}$ with each BER drop by 10 for BER $\leq 10^{-3}$. 
The required values of $\gamma$ in for BER of $10^{-3}$ and $10^{-6}$ are 11 aB and $21.5 \mathrm{~dB}$, respectively $(\mathrm{k}=1)$. The price paid to achieve the diversity gain 18 the increased complexity in the transmitter and the increased (doubled) bandwidth of the transmitted signal. The conventional binary PSK receiver is applied here.

\section{CONCLUSION}

A transmitter diversity scheme has been investigated for binary PSK mobile radio. In this scheme, the same binary information is used to modulate simultaneously three carriers uniformly spaced by the bit rate. The modulated carriers are tranemitted by separate antennas. A binary PSK recelver with a single antenna is used to modulate the signal. The BER performance was determined by theoretical analysis. The best performance occurs when the avaliable transmitter power is divided equally between the three carriers. Results show that the diveraity scheme has a gain of $12.5 \mathrm{~dB}$ and $33.5 \mathrm{~dB}$ at $\mathrm{BER}$ of $10^{-3}$ and $10^{-6}$, respectively.

\section{REFERENCES}

[1] T. Brenig, "Data Transmission for Mobile Radio," IBEE Trans. Veh. Technol., vol. VT-27, pp. 77-85, Aug. 1978.

[2] E. N. Gilbert, "Mobile Radio Diversity Reception," Bell Syst. Tech. J., vol. 48 , pp. 2473-2492, Oct. 1969. [3] W. C. Jakes, Jr. ,Ed., Microwave Mobile Communications. New York
Wiley, 1974 .

[4] J. D. Parsons et a1., "Diversity Techniques for Mobile Radio Faception," Radio and Electron. Bng., vol. 45, pp. 357-367, Ju1y 2975 .

[5] B. Ogose et al. "A Trangmitter Diversity for MSK with Two-Bit Differentil Detection," IEEE Trans. Veh, Technol., vol. $V T-33$, pp. 37-43, Feb. 1984 . 


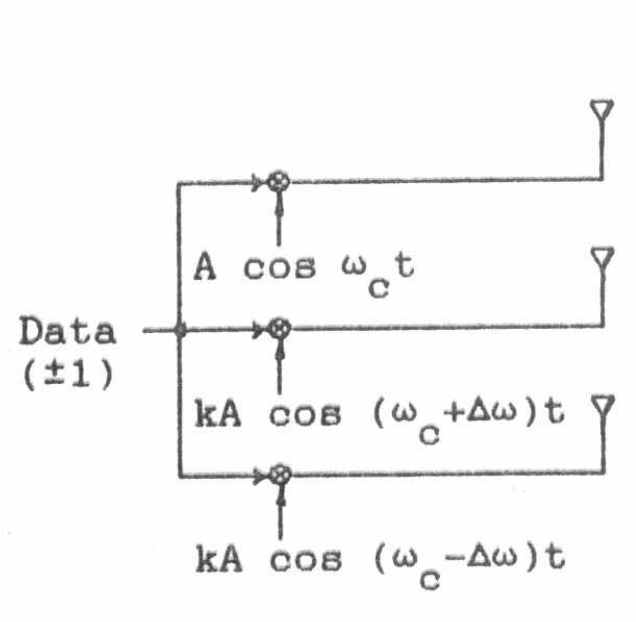

a) Transmitter

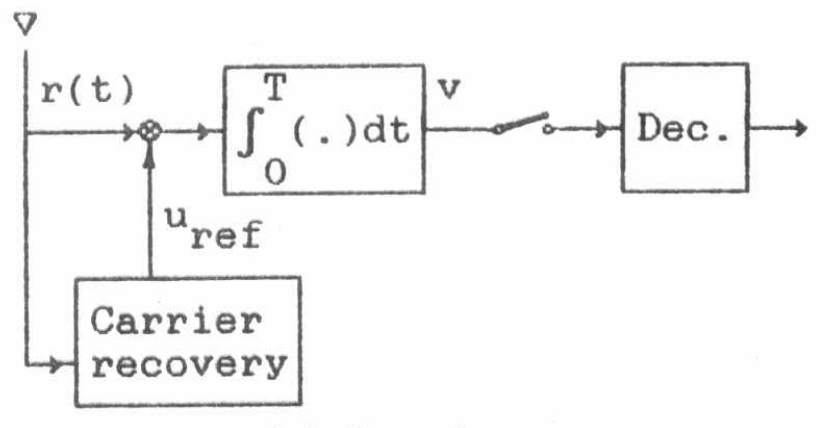

b) Receiver

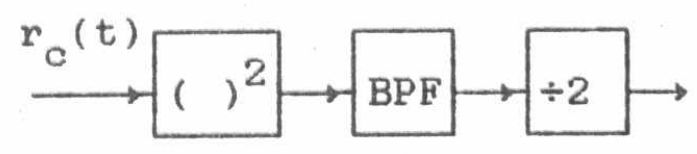

c) Carrier recovery

Fig. 1 System model

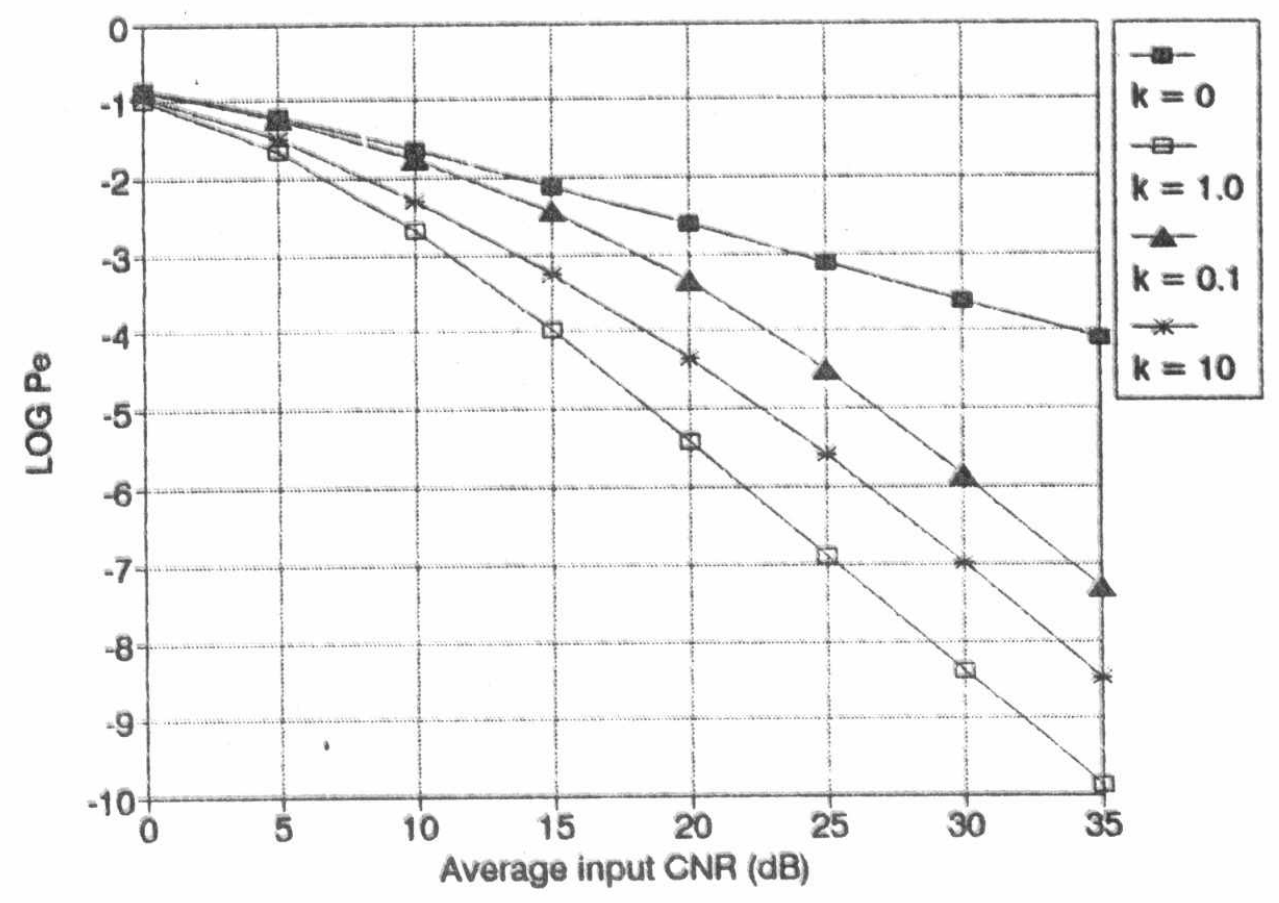

Fig. 2 BER Performance 